cancer. We designed the study to overcome certain limitations of earlier epidemiology studies.

Method The epidemiological and exposure assessment components of the study are coordinated by the University of Pittsburgh and the University of Illinois at Chicago, respectively. Our cohort comprises 12 manufacturing sites in the US and nine sites in Europe, and represents three companies, five countries (US, Austria, Germany, Sweden and UK) and multiple manufacturing processes and exposures. Statistical analyses will adjust external and internal mortality rate comparisons for potential co-exposures, including smoking histories obtained via a nested case-control study. The study will include separate and pooled analyses.

Results Our data collection effort identified two additional US study sites and additional subjects in the German and Swedish sites. Accordingly, our originally projected cohort size of 21 000 subjects has increased to 35508 (US-7005; Europe-33 508). Vital status tracing, cause of death determination and identification of subjects for the nested case-control study are ongoing.

Conclusions Our study will enable country-specific and pooled analyses of mortality rates and exposure-response relationships among workers from 21 study sites and the opportunity to compare and contrast findings across countries, sites, companies and/ or manufacturing processes and exposures involved in this global industry. We will detail progress to date on the US and combined epidemiological component of the study.

\section{MENTAL DISTRESS IN RELATION TO PSYCHOSOCIAL FACTORS AT WORK AMONG FAMILY PHYSICIANS}

1,2Vilija Malinauskiene. 'Lithuanian Sports University, Kaunas, Lithuania; 'Lithuanian University of Health Sciences, Kaunas, Lithuania

\subsection{6/oemed-2014-102362.303}

Objectives The study investigated the associations between mental health and job demands, control and social support at work and controlled for physical inactivity, sense of coherence and psychosocial factors in everyday life in a representative sample of Lithuanian family physicians.

Method With a response rate $89.2 \%$, a total of 323 family physicians filled in anonymous questionnaire on mental distress (GHQ-12) and psychosocial stressors at work and in everyday life, personal health resources (sense of coherence) and demographic variables. The statistical software SPSS 14.0 for Windows was used in the analysis. Associations were tested by way of multivariate logistic regression analysis.

Results The prevalence of mental distress was 40.2\% among family physicians and was positively associated with high job demands $(\mathrm{p}<0.001)$, low job control $(\mathrm{p}=0.009)$ and low social support at work $(\mathrm{p}=0.009)$. In a fully adjusted model controlling for age, gender, physical inactivity, sense of coherence, self-rated health, social relations and work-family interface, the adjusted OR for high job demands remained statistically significant with the $\mathrm{OR}=2.40 ; 1.40-4.12$, but for low job control it was 1.27 ; 95\% CI $0.74-2.19$, and for low social support at work - insignificant.

Conclusions Nearly half of the family physicians were mentally distressed. Psychosocial job characteristics, especially high job demands play an important role in poor mental health, as well as social relations, self-rated health and low sense of coherence. Health promotion strategies should be applied for this important occupation to diminish mental distress. WITH SHIFT WORK

${ }^{1}$ Pei-Chen Lin, ${ }^{2}$ Hsin-Chia Hung, ${ }^{3}$ Shung-Mei Pan, ${ }^{1,4}{ }^{4}$ Ming-Tsang Wu. ${ }^{1}$ Department of Public Health, College of Health Sciences, Kaohsiung Medical University, Kaohsiung City, Taiwan; ${ }^{2}$ Department of Nursing, Meiho University, Pingguang, Taiwan; ${ }^{3}$ Department of Nursing, Kaohsiung Medical University Hospital, Kaohsiung Medical University, Kaohsiung City, Taiwan; ${ }^{4}$ Center of Environmental and Occupational Medicine, Kaohsiung Municipal Hsiao-Kang Hospital, Kaohsiung City, Taiwan

\subsection{6/oemed-2014-102362.304}

Objectives To investigate the change of concentration of 8hydroxy-2'-deoxyguanosine $(8-\mathrm{OHdG})$ at different work time among shift work nurses.

Method A total of 51 nurses with regulated rotation shift in medical centre or district hospital. Urinary concentrations of 8-OHdG, a biomarker of oxidative DNA damage, were measured. According the rotation schedule, like 1-2 days off - 2 day shifts-2evenning shifts- 2night shifts, the urine was collected at 4 time points. The 2 workdays on the last day shift and last night shift of a shift course were selected and urine was collected at work before and finished work after on assigned workday, separately.

Results The urinary concentrations of $8-\mathrm{OHdG}$ at work after was significantly higher than at work before on the end of day shift (paired t test, p-value: 0.0363). The concentrations of 8OHdG between at work after and work before on the end of nigh shift were not significantly different (paired t test, p-value: 0.1673). Besides, there was no difference on the concentrations of 8 -OHdG at work after between a day shift and a night shift. We also found that the change of concentrations of 8-OHdG at a day shift was significantly different on work institution ( $p$ value: 0.0009 ).

Conclusions We found the more change of concentration of 8OHdG was at a day shift work then a night shift work, even night shift was as a risk factor of health.

Therefore, the change of concentration of 8 -OHdG was as a biomarker to respond the workload exposure.

\section{RISK OF LYMPHOMA AND OCCUPATIONAL EXPOSURE TO ORGANIC DUST}

lleana D'Andrea, Tinucia Nonne, Giannina Satta, Maria Valeria Flore, Luigi Lecca, Nicola Mascia, Tiziana Serra, Mariagrazia Zucca, Maria Grazia Ennas, Pierluigi Cocco. University of Cagliari, Monserrato, Cagliari, Italy

\subsection{6/oemed-2014-102362.305}

Objectives A medical history of allergy, and particularly asthma, has been associated with an inverse risk of non-Hodgkin's lymphoma (NHL). As occupational exposure to specific organic dusts is a risk factor for asthma, we explored risk of lymphoma and its major subtypes in relation to organic dusts.

Method In 1999-2004, 324 incident lymphoma cases and 464 population controls, frequency matched to cases by age and gender, were recruited among adult residents in Sardinia, Italy. Expert industrial hygienists assessed exposure to organic dust overall, and specific organic dusts. The odds ratio (OR) for lymphoma (all types) and its major subtypes, and its 95\% confidence interval, was calculated using unconditional logistic regression.

Results Exposure to organic dust in general was inversely associated with risk of lymphoma (all types) $(\mathrm{OR}=0.7,95 \% \mathrm{CI} 0.4$ 1.2), with a declining trend by duration and level of exposure. The inverse association was apparently more pronounced for 